\title{
UNIT ROOT TESTING IN THE PRESENCE OF INNOVATION VARIANCE BREAKS: A SIMPLE SOLUTION WITH INCREASED POWER
}

\section{STEVEN COOK}

Received 30 July 2001 and in revised form 5 March 2002

The Dickey-Fuller unit root test is known to suffer severe oversizing in the presence of innovation variance breaks. In this paper, forward and reverse Dickey-Fuller regressions are proposed as a means of correcting this size distortion. The results of Monte Carlo experimentation show such an approach to result in both satisfactory size properties and increased power relative to previously suggested solutions.

\section{Introduction}

The Dickey-Fuller (DF) test [4] is routinely employed in applied econometric analysis to examine the order of integration of economic time series. Recently, Kim et al. [9] have considered the properties of the DF test when applied to a unit root process, which experiences a break in innovation variance. This analysis is a welcome development, as in contrast to the huge literature on the behaviour of unit root tests in the presence of structural breaks in the level or trend of a time series (see, inter alia, Bai et al. [1]; Bai and Perron [2]; Banerjee et al. [3]; Perron [12, 13]), the impact of structural changes in variance has rarely been addressed, especially for integrated processes. (Wichern, Miller, and Hsu [14], Hsu [7], and Inclan [8] are cited by Kim et al. [9] as examples of the few instances where variance breaks have been considered in general circumstances. The only case cited where the impact of variance breaks has been considered in the context of integrated processes is Hamori and Tokihisa [6].) The evidence presented by Kim et al. [9] shows that when the break in variance takes the form of a large decrease early in the sample period, the DF can suffer severe size distortion. With a DF testing equation as 
in (1.1), the DF $\tau_{\mu}$ test is calculated as the $t$-statistic for null hypothesis $\mathrm{H}_{0}: \rho=1$,

$$
y_{t}=\mu+\rho y_{t-1}+\xi_{t}, \quad V\left(\xi_{t}\right)=\sigma^{2}
$$

Given (1.1), the $\tau_{\mu}$ test is subject to severe size distortion when there is a break in $\sigma^{2}$ early in the sample period. This spurious rejection of the unit hypothesis is in sharp contrast to the literature associated with Perron [12] where structural breaks are shown to cause $I(0)$ processes to appear I(1). In response to this, Kim et al. [9] develop an alternative Perron-style $[12,13]$ unit root test statistic based upon feasible modified generalised least squares, denoted by $t_{F}$. Having improved size properties in the presence of variance breaks, this test suffers however from low power in comparison to the $\tau_{\mu}$ test as the authors note.

In this paper, the properties of the $\mathrm{DF}_{\max }$ test of Leybourne [10] are examined in the presence of innovation variance breaks. Considering (1.1) above, the $\mathrm{DF}_{\max }$ test results from joint application of the $\tau_{\mu}$ test to both $\left\{y_{t}\right\}$ and $\left\{z_{t}\right\}$, where $z_{t}=y_{T-t+1}$ for $t=1, \ldots, T$, with the larger value obtained are denoted by $\mathrm{DF}_{\max }$. With early decreases in innovation variance for the forward regression becoming late increases in innovation variance for the reverse regression, the $\mathrm{DF}_{\max }$ test has an intuitive appeal, as neither late nor increasing breaks result in size distortion.

Using Monte Carlo simulation, the properties of the $\tau_{\mu}, \mathrm{DF}_{\max }$, and $t_{F}$ tests are examined in the presence of innovation variance breaks. Crucially, it is found that in the majority of the cases considered, the $\mathrm{DF}_{\max }$ has a clear power advantage over the $t_{F}$ test, while exhibiting similar size.

\section{Monte Carlo simulation}

\subsection{Experimental design}

To allow a direct comparison with the results of Kim et al. [9] for the $t_{F}$ test, the following data generation process (DGP) was employed:

$$
\begin{aligned}
y_{t} & =\rho y_{t-1}+\varepsilon_{t}, \quad t=1, \ldots, T, \\
\varepsilon_{t} & =\sigma_{t} \eta_{t}, \\
\eta_{t} & \sim \text { i.i.d. } N(0,1), \\
\sigma_{t} & = \begin{cases}\sigma_{1} & \text { for } t \leqslant \tau^{*} T, \\
\sigma_{2} & \text { for } t>\tau^{*} T,\end{cases}
\end{aligned}
$$


where $\tau^{*}$ represents the break fraction determining the point at which there is an abrupt change in the error variance. (The error series $\left\{\eta_{t}\right\}$ was generated using pseudo i.i.d. $N(0,1)$ random numbers from the RNDNS procedure in the Gauss programming language version 3.2.13, with the initial value $\left(y_{0}\right)$ set equal to zero. All experiments were performed over 40,000 replications with the first 100 observations created discarded to remove the influence of initial conditions.)

Kim et al. [9] show size distortion of the $\tau_{\mu}$ test to depend upon the size of the decrease in variance and the time at which it occurs. Denoting the break size $\left(\sigma_{2} / \sigma_{1}\right)$ as $\delta$, the values $\delta \in\{0.25,0.4,0.6,0.8,1.0\}$ are considered, with $\delta=1$ denoting no break in variance. Following Kim et al. [9], the values $\tau^{*} \in\{0.2,0.4,0.6,0.8\}$ are chosen for the break fractions. Similarly, two sample sizes are considered with $T \in\{100,200\}$. For each of the experimental designs, the $\tau_{\mu}$ and $\mathrm{DF}_{\max }$ tests are estimated, with the results obtained compared to those of Kim et al. [9] for the $t_{F}$ test. To consider the sizes of the unit root tests in the presence of variance breaks, the value $\rho=1$ was imposed in the DGP. To assess the power of the tests, near integrated processes are considered. The values chosen for $\rho$ in these cases are $\rho=0.9$ for $T=100$, and $\rho=0.95$ for $T=200$. (Alternative values of $\rho$, and unit root tests containing both intercept and trend terms were also considered. As the results obtained for these additional experiments were similar to those presented here, they have been omitted in the interests of brevity. However, the results are available from the author upon request.) Empirical rejection frequencies in all cases are calculated at the 5\% nominal level of significance $(\alpha=0.05)$, with the $\tau_{\mu}$ and $\mathrm{DF}_{\max }$ tests employing critical values from Fuller [5] and Leybourne [10], respectively.

\subsection{Results}

The results of the size experiments are presented in Table 2.1. The results show that the $\tau_{\mu}$ test can suffer severe size distortion across a range of values of $\delta$ and $\tau^{*}$, with an empirical size of $39.3 \%$ observed for $\left\{\delta, \tau^{*}, T\right\}=\{0.25,0.2,100\}$. Although the $\mathrm{DF}_{\max }$ test can also experience oversizing for large breaks early in the sample, for more moderate breaks or later breaks, it has better size properties than the $t_{F}$ test. This is illustrated in Figure 2.1 where results for the rival tests are presented for $\{\delta, T\}=\{0.6,100\}$. It should also be recognised that the values of $\delta$ considered relate to the standard deviations of the innovations, not their variances. Therefore, $\delta=0.6$ relates to a change in variance by a factor of approximately 3 , with the extreme value $\delta=0.25$ indicating the case where $\sigma_{1}^{2}=16 \sigma_{2}^{2}$. It can therefore be questioned how much weight should be attached to the extreme cases where $\delta$ takes such small values. (The change in variance can be considered in terms of the vari- 


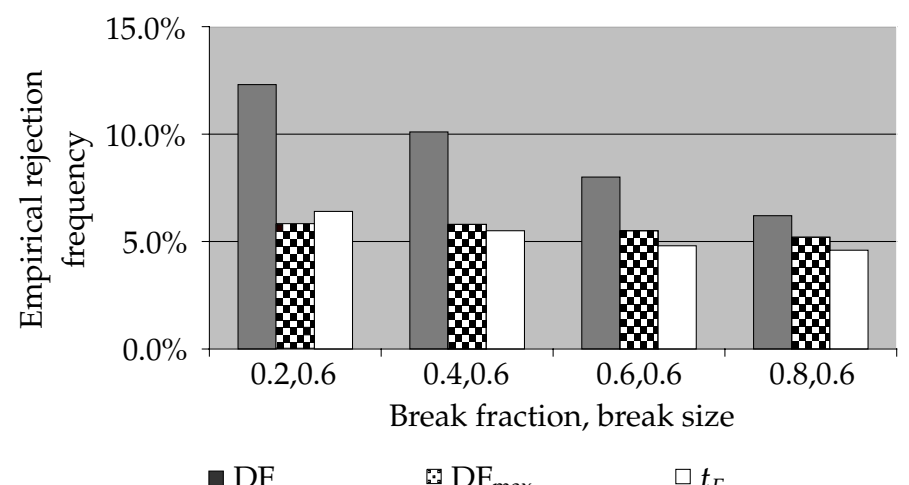

FIGURE 2.1. Empirical size for $\alpha=0.05$ and $T=100$.

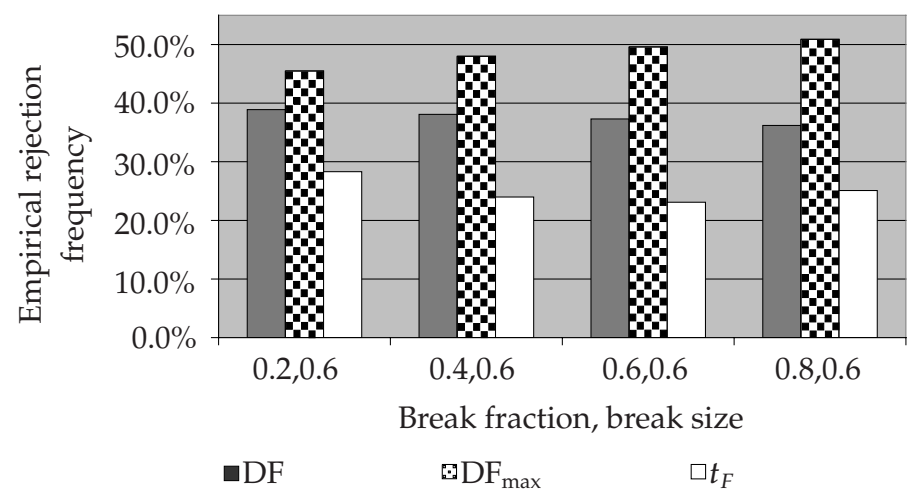

FigURE 2.2. Power for $\alpha=0.05, T=100$, and $\rho=0.9$.

ance ratio test. For the relatively large degrees of freedom considered here, the variance ratio test would easily reject the null of constant variances given a calculated value of 3 . This indicates that a relatively large shift is being considered. A value of 16 would be viewed as extremely high.)

Considering the power results contained in Table 2.2, it can be seen that the $t_{F}$ test has the lowest power of the tests in the vast majority of experiments, the exceptions being when the largest possible break occurs at the earliest possible point. When more moderate, plausible breaks in variance are considered, the $\mathrm{DF}_{\max }$ test has a clear power advantage over the $t_{F}$ test. Figure 2.2 illustrates this, displaying results for $(T, \delta, \rho)=(100,0.6,0.9)$, where the $\mathrm{DF}_{\max }$ test has $61 \%$ more power than the $t_{F}$ test. 
TABLe 2.1. Empirical size $(\alpha=0.05)$.

\begin{tabular}{lllllll}
\hline$\tau^{*}$ & & $\delta=1.0$ & $\delta=0.8$ & $\delta=0.6$ & $\delta=0.4$ & $\delta=0.25$ \\
\hline \multirow{4}{*}{0.2} & $\tau_{\mu}$ & 0.050 & 0.073 & 0.123 & 0.234 & 0.393 \\
& $\mathrm{DF}_{\max }$ & 0.050 & 0.051 & 0.058 & 0.080 & 0.132 \\
& $t_{F}$ & 0.053 & 0.062 & 0.064 & 0.065 & 0.066 \\
\hline \multirow{2}{*}{0.4} & $\tau_{\mu}$ & $\ldots$ & 0.068 & 0.101 & 0.165 & 0.239 \\
& $\mathrm{DF}_{\max }$ & $\ldots$ & 0.051 & 0.058 & 0.077 & 0.105 \\
& $t_{F}$ & $\ldots$ & 0.054 & 0.055 & 0.058 & 0.059 \\
\hline \multirow{3}{*}{0.6} & $\tau_{\mu}$ & $\ldots$ & 0.062 & 0.080 & 0.105 & 0.131 \\
& $\mathrm{DF}_{\max }$ & $\ldots$ & 0.051 & 0.055 & 0.066 & 0.076 \\
& $t_{F}$ & $\ldots$ & 0.047 & 0.048 & 0.056 & 0.057 \\
\hline \multirow{3}{*}{0.8} & $\tau_{\mu}$ & $\ldots$ & 0.056 & 0.062 & 0.070 & 0.074 \\
& $\mathrm{DF}_{\max }$ & $\ldots$ & 0.051 & 0.052 & 0.054 & 0.055 \\
& $t_{F}$ & $\ldots$ & 0.046 & & & \\
\hline
\end{tabular}

(a) $T=100$.

\begin{tabular}{lllllll}
\hline$\tau^{*}$ & & $\delta=1.0$ & $\delta=0.8$ & $\delta=0.6$ & $\delta=0.4$ & $\delta=0.25$ \\
\hline \multirow{4}{*}{0.2} & $\tau_{\mu}$ & 0.049 & 0.072 & 0.120 & 0.229 & 0.390 \\
& $\mathrm{DF}_{\max }$ & 0.050 & 0.051 & 0.058 & 0.082 & 0.138 \\
& $t_{F}$ & 0.052 & 0.055 & 0.055 & 0.057 & 0.058 \\
\hline \multirow{3}{*}{0.4} & $\tau_{\mu}$ & $\ldots$ & 0.066 & 0.099 & 0.162 & 0.237 \\
& $\mathrm{DF}_{\max }$ & $\ldots$ & 0.051 & 0.059 & 0.081 & 0.110 \\
& $t_{F}$ & $\ldots$ & 0.053 & 0.052 & 0.052 & 0.050 \\
\hline \multirow{3}{*}{0.6} & $\tau_{\mu}$ & $\ldots$ & 0.059 & 0.077 & 0.105 & 0.129 \\
& $\mathrm{DF}_{\max }$ & $\ldots$ & 0.051 & 0.057 & 0.068 & 0.078 \\
& $t_{F}$ & $\ldots$ & 0.048 & 0.050 & 0.051 & 0.053 \\
\hline \multirow{3}{*}{0.8} & $\tau_{\mu}$ & $\ldots$ & 0.053 & 0.060 & 0.068 & 0.074 \\
& $\mathrm{DF}_{\max }$ & $\ldots$ & 0.050 & 0.051 & 0.054 & 0.055 \\
& $t_{F}$ & $\ldots$ & 0.048 & 0.049 & 0.052 & 0.053 \\
\hline
\end{tabular}

(b) $T=200$. 
Unit roots and variance breaks

TABLe 2.2. Power $(\alpha=0.05)$.

\begin{tabular}{lllllll}
\hline$\tau^{*}$ & & $\delta=1.0$ & $\delta=0.8$ & $\delta=0.6$ & $\delta=0.4$ & $\delta=0.25$ \\
\hline \multirow{3}{*}{0.2} & $\tau_{\mu}$ & 0.339 & 0.354 & 0.389 & 0.452 & 0.508 \\
& $\mathrm{DF}_{\max }$ & 0.512 & 0.487 & 0.455 & 0.428 & 0.408 \\
& $t_{F}$ & 0.250 & 0.260 & 0.283 & 0.347 & 0.472 \\
\hline \multirow{2}{*}{0.4} & $\tau_{\mu}$ & $\ldots$ & 0.352 & 0.381 & 0.422 & 0.448 \\
& $\mathrm{DF}_{\max }$ & $\ldots$ & 0.496 & 0.480 & 0.467 & 0.457 \\
& $t_{F}$ & $\ldots$ & 0.243 & 0.240 & 0.287 & 0.395 \\
\hline \multirow{3}{*}{0.6} & $\tau_{\mu}$ & $\ldots$ & 0.351 & 0.373 & 0.395 & 0.408 \\
& $\mathrm{DF}_{\max }$ & $\ldots$ & 0.504 & 0.496 & 0.491 & 0.488 \\
& $t_{F}$ & $\ldots$ & 0.244 & 0.231 & 0.262 & 0.338 \\
\hline \multirow{3}{*}{0.8} & $\tau_{\mu}$ & $\ldots$ & 0.349 & 0.362 & 0.375 & 0.382 \\
& $\mathrm{DF}_{\max }$ & $\ldots$ & 0.510 & 0.509 & 0.508 & 0.505 \\
& $t_{F}$ & $\ldots$ & 0.241 & 0.251 & 0.280 & 0.323 \\
\hline
\end{tabular}

(a) $\rho=0.9, T=100$.

\begin{tabular}{clccccc}
\hline$\tau^{*}$ & & $\delta=1.0$ & $\delta=0.8$ & $\delta=0.6$ & $\delta=0.4$ & $\delta=0.25$ \\
\hline \multirow{4}{*}{0.2} & $\tau_{\mu}$ & 0.330 & 0.346 & 0.380 & 0.447 & 0.508 \\
& $\mathrm{DF}_{\max }$ & 0.509 & 0.483 & 0.450 & 0.425 & 0.409 \\
& $t_{F}$ & 0.260 & 0.272 & 0.300 & 0.370 & 0.489 \\
\hline \multirow{4}{*}{0.4} & $\tau_{\mu}$ & $\ldots$ & 0.346 & 0.376 & 0.417 & 0.445 \\
& $\mathrm{DF}_{\max }$ & $\ldots$ & 0.493 & 0.479 & 0.467 & 0.457 \\
& $t_{F}$ & $\ldots$ & 0.247 & 0.252 & 0.289 & 0.415 \\
\hline \multirow{3}{*}{0.6} & $\tau_{\mu}$ & $\ldots$ & 0.343 & 0.364 & 0.388 & 0.401 \\
& $\mathrm{DF}_{\max }$ & $\ldots$ & 0.501 & 0.495 & 0.490 & 0.485 \\
& $t_{F}$ & $\ldots$ & 0.247 & 0.246 & 0.284 & 0.370 \\
\hline & $\tau_{\mu}$ & $\ldots$ & 0.340 & 0.353 & 0.369 & 0.378 \\
0.8 & $\mathrm{DF}_{\max }$ & $\ldots$ & 0.507 & 0.507 & 0.506 & 0.505 \\
& $t_{F}$ & $\ldots$ & 0.260 & 0.273 & 0.292 & 0.333 \\
\hline
\end{tabular}

(b) $\rho=0.95, T=200$. 


\section{Conclusion}

In this paper, recent research on the testing of unit roots in the presence of breaks in innovation variance has been extended. The results presented show that although the $t_{F}$ test of Kim et al. [9] removes size distortion in the presence of extreme variance breaks, for less extreme cases the $\mathrm{DF}_{\max }$ test of Leybourne [10] has similar, and sometimes better, size properties. It has also been seen that the low power of the $t_{F}$ test is not shared by the $\mathrm{DF}_{\max }$ test, which has high power against near integrated alternatives over a range of plausible variance breaks. The size and power analyses therefore suggest that the $\mathrm{DF}_{\max }$ test is of practical importance in presence of innovation variance breaks, a finding which contrasts with the suggestion of Leybourne et al. [11] for the case of breaks in level or drift.

\section{Acknowledgment}

The author is grateful to an anonymous referee for comments which have led to a significant improvement in the presentation of this paper.

\section{References}

[1] J. Bai, R. L. Lumsdaine, and J. H. Stock, Testing for and dating common breaks in multivariate time series, Rev. Econom. Stud. 65 (1998), no. 3, 395-432.

[2] J. Bai and P. Perron, Testing for and estimation of multiple structural changes, Econometrica 66 (1998), 47-79.

[3] A. Banerjee, R. Lumsdaine, and J. Stock, Recursive and sequential tests of the unit root and trend break hypotheses: Theory and international evidence, J. Bus. Econom. Statist. 10 (1992), 271-287.

[4] D. A. Dickey and W. A. Fuller, Distribution of the estimators for autoregressive time series with a unit root, J. Amer. Statist. Assoc. 74 (1979), no. 366, part $1,427-431$.

[5] W. A. Fuller, Introduction to Statistical Time Series, John Wiley \& Sons, New York, 1976.

[6] S. Hamori and A. Tokihisa, Testing for a unit root in the presence of a variance shift, Econom. Lett. 57 (1997), no. 3, 245-253.

[7] S. Hsu, Tests for variance shift at an unknown time point, J. Appl. Statist. 26 (1977), 279-284.

[8] C. Inclan, Detection of multiple changes of variance using posterior odds, J. Bus. Econom. Statist. 11 (1993), 289-300.

[9] T.-H. Kim, S. Leybourne, and P. Newbold, Unit root tests with a break in innovation variance, mimeo, University of Nottingham, 2000.

[10] S. Leybourne, Testing for unit roots using forward and reverse Dickey-Fuller regressions, Oxford Bulletin of Economics and Statistics 57 (1995), 559-571.

[11] S. Leybourne, T. Mills, and P. Newbold, Spurious rejections by Dickey-Fuller tests in the presence of a break under the null, J. Econometrics 87 (1998), 191203. 
240 Unit roots and variance breaks

[12] P. Perron, The great crash, the oil price shock and the unit root hypothesis, Econometrica 57 (1989), 1361-1401.

[13] _ Testing for a unit root in time series with a changing mean, J. Bus. Econom. Statist. 8 (1990), 153-162.

[14] D. Wichern, R. Miller, and D. Hsu, Changes of variance in first order autoregressive time series models with an application, J. Appl. Statist. 25 (1976), 248-256.

Steven Cook: Department of Economics, University of Wales Swansea, Singleton Park, Swansea SA2 8PP, Wales, UK

E-mail address: s.cook@swan.ac.uk 


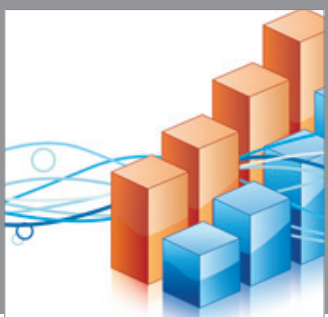

Advances in

Operations Research

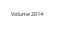

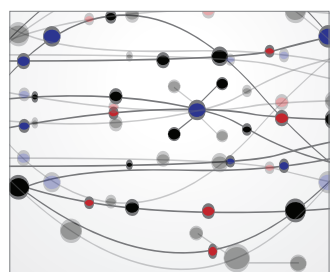

\section{The Scientific} World Journal
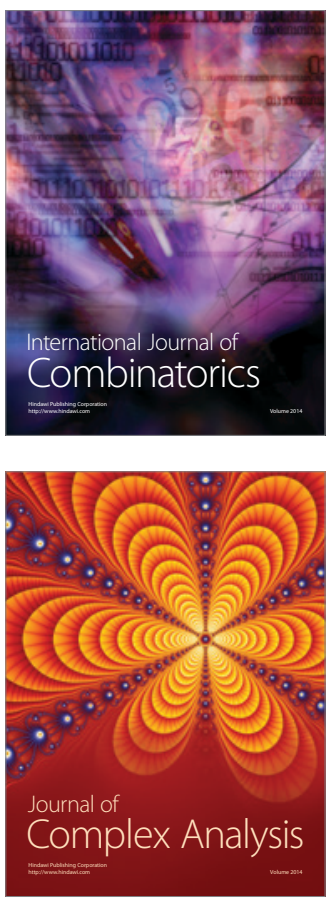

International Journal of

Mathematics and

Mathematical

Sciences
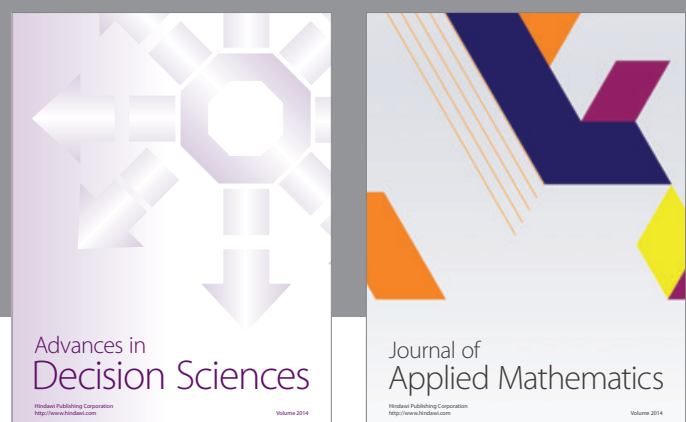

Journal of

Applied Mathematics
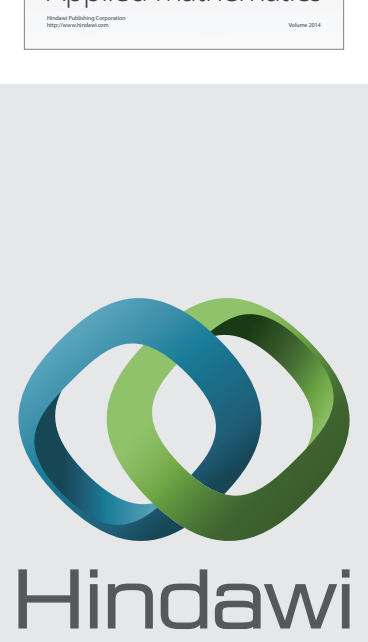

Submit your manuscripts at http://www.hindawi.com
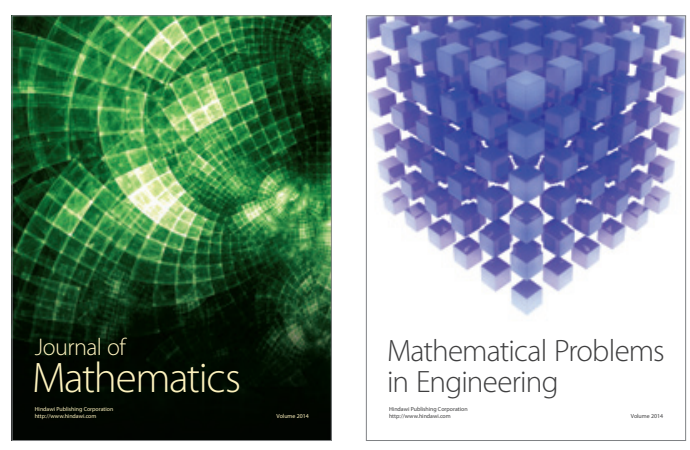

Mathematical Problems in Engineering
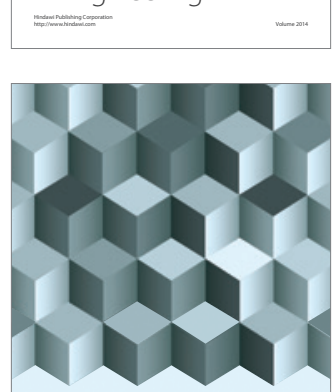

Journal of

Function Spaces
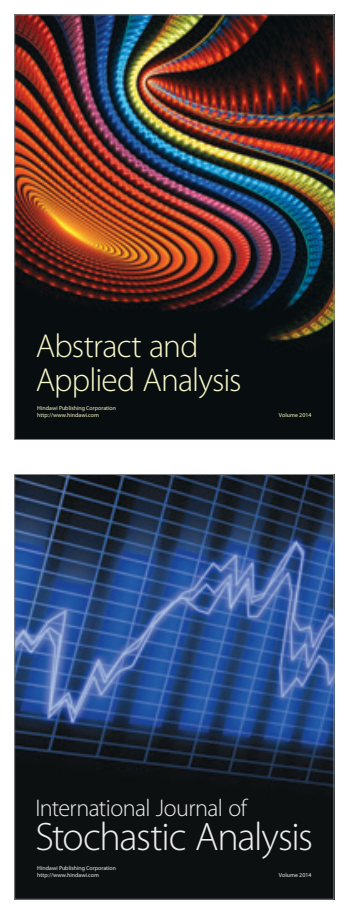

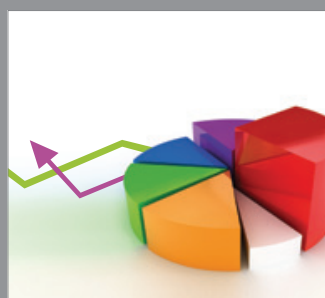

ournal of

Probability and Statistics

Promensencen
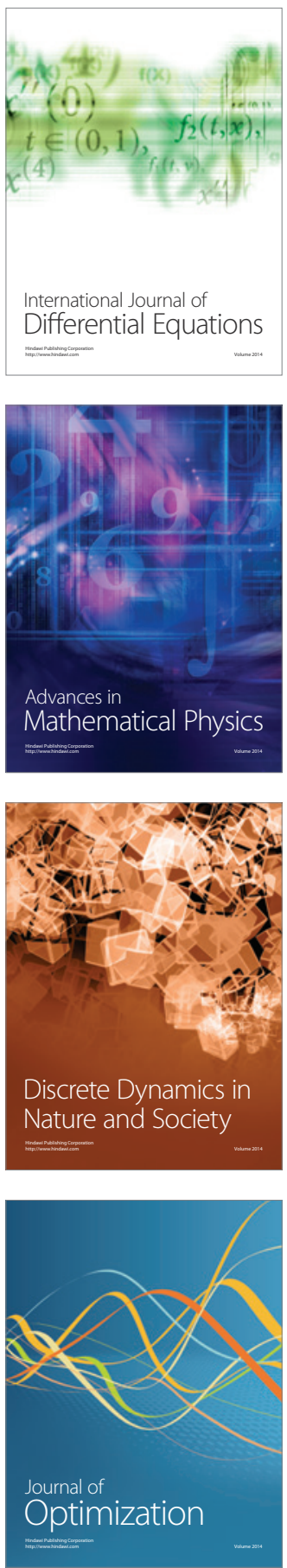